\title{
Evaluation of applied potential tomography as a new non-invasive gastric secretion test
}

\author{
A J BAXTER, Y F MANGNALL, E H LOJ, B BROWN, D C BARBER, \\ A G JOHNSON, AND N W READ \\ From the Sub-Department of Human Gastrointestinal Physiology and Nutrition and Department of Surgery, \\ University of Sheffield, Royal Hallamshire Hospital, Sheffield
}

SUmmary Applied potential tomography (APT) is a new, non-invasive technique that can yield sequential images of changes in the resistivity of gastric contents. Studies were performed to investigate the application of APT to measure gastric acid secretion. Experiments in 20 normal volunteers showed that changes in gastric resistivity were closely correlated with changes in the volume $(r=0 \cdot 80)$, the acidity $(r=0 \cdot 83)$ and the total conductivity of gastric contents $(r=0 \cdot 87)$. Studies in 13 patients referred for a pentagastrin test showed that changes in gastric resistivity before pentagastrin were closely correlated with basal acid output measured on a separate occasion $(\mathbf{r}=0.85, p<0.001)$, while changes in gastric resistivity after pentagastrin were correlated with maximal acid output $(r=0 \cdot 58, p<0 \cdot 05)$. Ingestion of alcohol by six normal subjects decreased gastric resistivity markedly, probably due to alcohol induced gastric acid secretion as it was prevented by cimetidine. Applied potential tomography is a safe non-invasive method of measuring gastric acid secretion. The equipment is simple to use, and the test is comfortable and acceptable to patients.

Gastric acid secretion is usually measured by aspirating gastric contents through a nasogastric tube before and after an injection of pentagastrin. ${ }^{.}$Because patients often find intubation stressful and uncomfortable, there is a need for a non-invasive method for measuring gastric acid secretion.

Several non-invasive tests have been evaluated. The measurement in the urine of cation exchange dyes, that are split to yield absorbable products in an acidic environment, provide only a qualitative index of acid secretion..$^{2-4}$ Scintigraphic measurement of the accumulation of ${ }^{\omega_{\mathrm{m}} \mathrm{T}} \mathrm{Tc}$ in the stomach after an intravenous injection is more accurate,${ }^{56}$ but involves the use of radioactive tracers and technetium accumulation may reflect mucosal blood flow and stomach surface area rather than gastric acid output.?

Applied potential tomography is a new noninvasive, non-radioactive technique that can generate tomographic images of the resistivity of gastric contents, ${ }^{x-11}$ using electrodes placed around

Address for correspondence: Dr N W Read, Sub-Department of Human Gastrointestinal Physiology and Nutrition, K Floor, Royal Hallamshire Hospital, Sheffield S10 2JF

Received for publication 8 June 1988. the upper abdomen. This technique is simple to operate, quite comfortable for the patient and can provide an accurate index of gastric emptying, but only if the secretion of gastric acid has been inhibited with $\mathrm{H}_{2}$ receptor antagonists." This latter observation suggests that acid secretion could alter the resistivity of gastric contents. The aim of this study was to assess the possible application of APT as a non-invasive test for gastric secretion, by determining the correlation between resistivity changes measured by APT and gastric acidity.

\section{Methods}

\section{SUBJECTS}

Studies were carried out on a total of 20 fasted healthy volunteers, aged between 18-27 years (11 men, nine women) and on eight male and five female patients with peptic ulcer disease aged between 35 and 69 years, referred for pentagastrin tests of gastric function. All subjects gave written informed consent for the study and the protocol was approved by the Southern District Ethical Sub-Committee of the Sheffield Area Health Authority (Teaching). 
Gastric resistivity was measured through 16 electrodes evenly placed around the abdomen at the level of the eighth costal cartilage (a plane transecting the gastric fundus or body). An alternating current of $1 \mathrm{~mA}$ at $50 \mathrm{Khz}$ was passed between two adjacent electrodes (the drive electrodes) and the potential gradients at the remaining 13 pairs were measured. Each pair of electrodes in turn acted as drive electrodes. This constituted one cycle which lasted approximately $100 \mathrm{~ms}^{4-11}$ One hundred and fifty cycles were summated to form one data set.

An initial data set was recorded, and subsequent data sets were back projected against the initial set to produce a cross sectional image of the change in distribution of resistivity in the area of the electrodes. Sequential images were generated at one minute intervals. The images incorporated changes in resistivity occurring at least $4 \mathrm{~cm}$ above and below the electrode plane. To analyse the data, the region of the stomach was outlined with a cursor and values for the changes in resistivity within that region were calculated.

\section{VALIDATION IN HEALTHY SUBJECTS}

Experiments were carried out to measure the resistivity changes after the sequential introduction of either a series of solutions of varying volume and the same $\mathrm{pH}$ or a series of solutions of the same volume and varying $\mathrm{pH}$. Gastric solutions were made up from a stock solution of $100 \mathrm{mmol}$ hydrochloric acid diluted with $154 \mathrm{mmol}$ saline.

Each subject was intubated with a double lumen sump tube (Mallinckrodt, Athlone, Ireland) which was positioned in the most dependent part of the stomach. The stomach was washed out at least five times by injection of $20 \mathrm{ml}$ isotonic saline followed by immediate aspiration. A reference image was then taken.

The effect of $\mathrm{pH}$ on gastric resistivity was investigated in eight volunteers. Solutions containing 5, 10, 25,50 , and $100 \mathrm{mmol} \mathrm{HCl}$ were used. After washing the stomach out with $20 \mathrm{ml}$ of $154 \mathrm{mmol}$ saline, $50 \mathrm{ml}$ of the test solution was injected through the intragastric tube. Two minutes later, the test solution was aspirated and the stomach was washed out with saline before the next test solution was introduced. Solutions were introduced in order of increasing hydrogen ion concentration in order to reduce the effect of contamination by the previous solution. The region of interest was outlined from the image obtained immediately after the infusion of $50 \mathrm{ml} 100$ mmol hydrochloric acid.

The effect of volume of acid on gastric resistivity was investigated in six volunteers. Different volumes of solutions containing $25 \mathrm{mmol}$ hydrochloric acid $(50,100$, and $140 \mathrm{ml})$ and $50 \mathrm{mmol}$ hydrochloric acid
$(25,50$, and $70 \mathrm{ml})$ were serially injected into the stomach. The stomach was washed out with saline before the introduction of each solution, as described above. The effect of varying the conductivity (calculated from the relative ionic mobility and number of moles of each ion present) of the solutions introduced into the stomach on changes in APT was measured in the same volunteers. Two hundred and fifty millilitres $5 \mathrm{mmol}, 250$ and $125 \mathrm{ml} 10 \mathrm{mmol}, 100 \mathrm{ml} 35$ $\mathrm{mmol}$, and 25 and $35 \mathrm{ml} 100 \mathrm{mmol}$ hydrochloric acid were serially injected into the stomach as before. The region of interest was outlined from the image obtained immediately after infusion of $250 \mathrm{ml} 10$ mmol hydrochloric acid.

The changes in resistivity immediately after infusion of each solution were expressed as a percentage of the maximum changes in resistivity, obtained immediately after infusion of $50 \mathrm{ml}$ of $100 \mathrm{mmol} \mathrm{HCl}$ ( $\mathrm{pH}$ experiments), and $140 \mathrm{ml}$ of $25 \mathrm{mmol} \mathrm{HCl}$ and 70 $\mathrm{ml}$ of $50 \mathrm{mmol} \mathrm{HCl}$ (volume experiments), and 250 $\mathrm{ml} 10 \mathrm{mmol}$ (conductivity experiments).

VALIDATION IN PATIENTS

Thirteen patients participated in the study. Two studies were carried out in random order and separated by at least a week. All were fasted for 12 hours and had not taken any medication for 48 hours. On one occasion a pentagastrin test was performed. Patients were intubated with a double lumen sump tube which was positioned with its tip in the most dependent part of the stomach. Gastric acid was collected by continuous aspiration. Four 15 minute samples were collected and their volume noted. Acid concentration was measured by titration to $\mathrm{pH} 7$ using an autotitrator (Radiometer, Copenhagen). Acid output was calculated from volume and acid concentration for each sample and the results summed to give basal acid output (BAO). Subjects were then given $6 \mu \mathrm{g} / \mathrm{kg}$ pentagastrin im (Peptavlon, ICI, Macclesfield, Cheshire) and gastric acid collected for a further hour. Six 10 minute samples were collected. Acid output was calculated for each period as before and summed to give maximum acid output (MAO). On another occasion, the patients were not intubated and gastric resistivity was measured using APT, before and after the same dose of pentagastrin. Images were collected at one minute intervals under basal conditions for 30 minutes. The patient was then given $6 \mu \mathrm{g} / \mathrm{kg}$ pentagastrin im and images were collected for a further 60 minutes. Finally, a drink consisting of a beef stock cube (Oxo, Brooke Bond, Croydon, England) dissolved in 500 $\mathrm{ml}$ water warmed to $37^{\circ} \mathrm{C}$ was administered in order to identify the gastric region of interest. A fixed size region of interest was outlined, thus allowing comparison between patients of actual resistivity changes. 


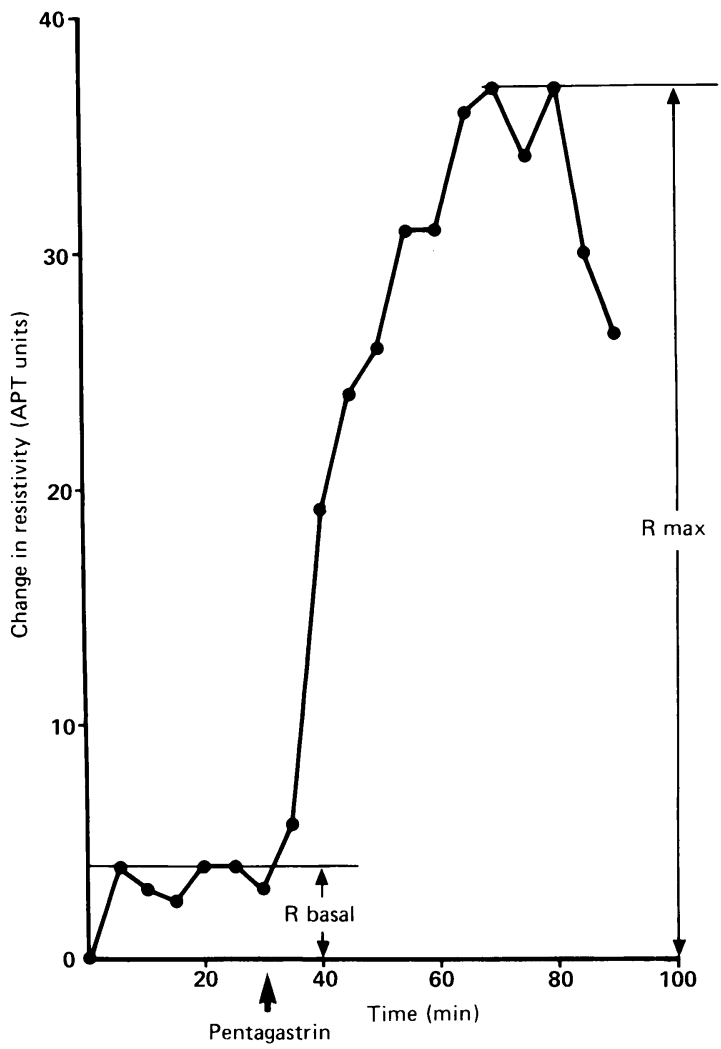

Fig. 1 Typical example of the profile of changes in gastric resistivity obtained by $A P T$ in a patient before and after administration of pentagastrin.

The maximum change in resistivity that occurred in each subject during the basal half hour ( $\mathrm{R}$ basal) was compared with the basal acid output for that subject, while the maximum change in resistivity that occurred in each subject during the whole study ( $R$ maximum) was compared with the maximal acid output (Fig. 1).

\section{EFFECT OF AN ALCOHOLIC DRINK ON GASTRIC} RESISTIVITY

Experiments were undertaken to measure the change in gastric resistivity in fasted volunteers after a drink of alcohol.

Six volunteers were fasted for 12 hours before the study. The APT images were collected at minute intervals for 30 minutes to provide a basal record. The subject then drank $30 \mathrm{ml}$ sherry (Old England Medium British Sherry, J E Mather \& Sons Ltd) and APT images were collected for a further 50 minutes. At the end of the study, the subject drank $300 \mathrm{ml}$ of Oxo solution to show the position of the stomach.

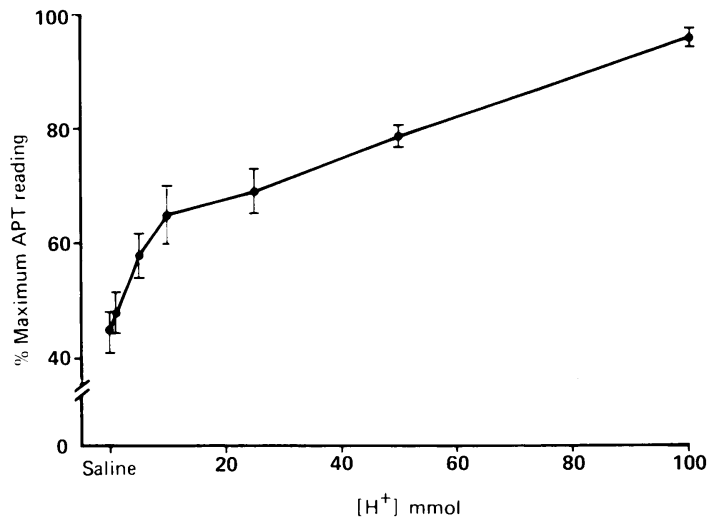

Fig. 2 Changes in gastric resistivity obtained immediately after injection of $50 \mathrm{ml}$ solutions of increasing hydrochloric acid concentration into the stomach of eight normal volunteers. Results (mean (SE)) are expressed as a percentage of the maximum changes obtained with the largest acid concentrations.

The region of interest was outlined from the image obtained immediately after ingestion of the Oxo drink, and the results expressed as a percentage of the maximum APT reading obtained after the drink of Oxo.

The experiment was repeated in three volunteers who had been given $800 \mathrm{mg}$ cimetidine (Tagamet, SK\&F, Welwyn, UK) one and a half hours before the start of the study to suppress acid secretion.

STATISTICAL ANALYSIS

The relationships between gastric resistivity and gastric acidity values were compared using linear regression analysis. A paired $t$ test was used to compare results obtained after infusion of saline into the stomach.

\section{Results}

VALIDATION IN VOLUNTEERS

Increasing the acid concentration of solutions of identical volume caused sequential reduction in gastric resistivity (Fig. 2), and there was a significant correlation between hydrogen ion concentration and changes in resistivity $(\mathrm{r}=0.83 ; \mathrm{p}<0.001)$. Increasing the volume of solutions containing the same concentrations of hydrogen ion also caused sequential reduction in gastric resistivity (Fig. 3). Significant linear correlations were observed between the changes in resistivity and the volumes of solutions containing $25 \mathrm{mmol}(\mathrm{r}=0.92, \mathrm{p}<0.001)$ and $50 \mathrm{mmol}$ hydrochloric acid $(\mathrm{r}=0.77 ; \mathrm{p}<0.001)$. There was a significant correlation between changes in gastric resistivity as determined by APT and the changes in 


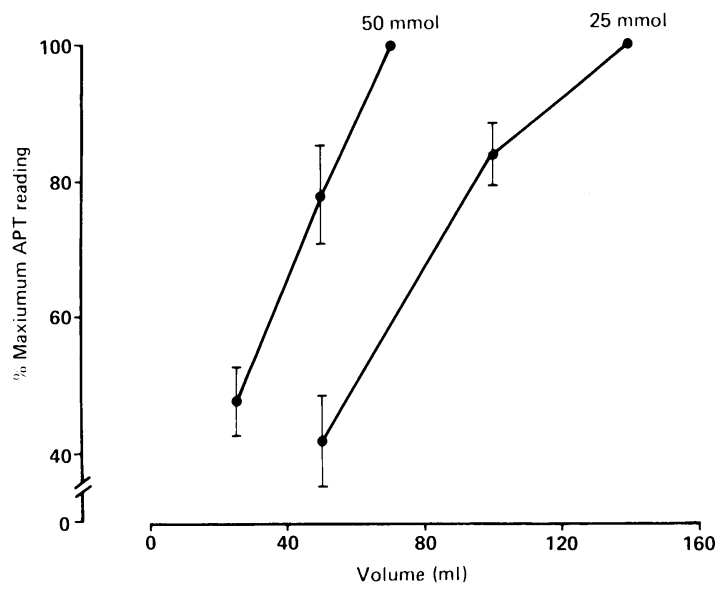

Fig. 3 Changes in gastric resistivity obtained immediately after injection of increasing volumes of $50 \mathrm{mmol}$ and 25 mmol hydrochloric acid into the stomach of six normal volunteers. The results (mean (SE)) are expressed as a percentage of the maximum changes obtained with the largest volumes.

the conductivity of the solutions introduced into the stomach $(r=0.87 ; p<0 \cdot 001)$ (Fig. 4).

There was no significant difference between APT readings obtained by infusion of $50 \mathrm{ml}$ saline into the stomach before and after infusion of 12 different test solutions containing acid $((25 \cdot 3)(2 \cdot 1) v 26 \cdot 1(2 \cdot 9) \%$ $\max$ APT; $p>0 \cdot 05)$. Hence there was no significant

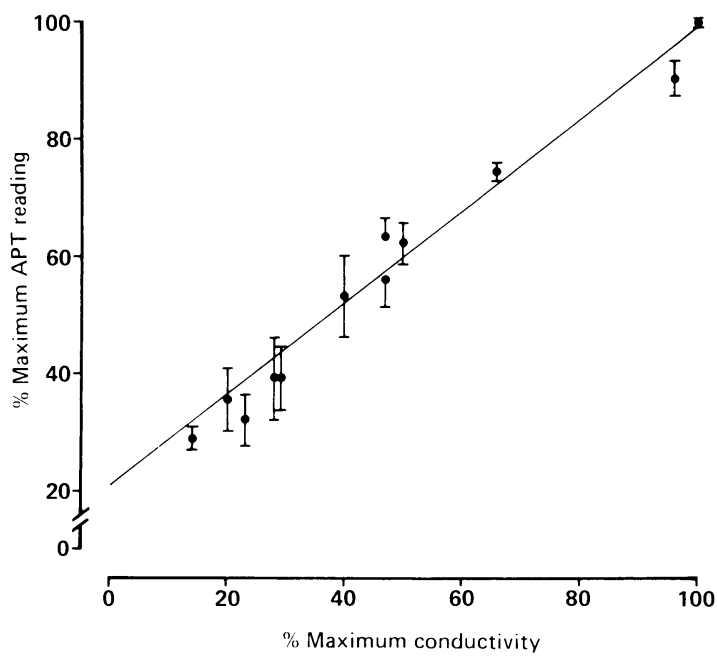

Fig. 4 Relationship between change in gastric resistivity, obtained after injection of solutions of differing volume and acidity into the stomach of six normal volunteers, and the conductivity of the solutions. Results (mean (SE)) are expressed as a percentage of the maximum change obtained with the solution of greatest conductivity. consistent baseline variation in APT readings during the experiment. (Mean (SE)).

VALIDATION IN PATIENTS

The change in resistivity that occurred during the basal period ( $\mathrm{R}$ basal) was correlated with the basal acid output $(r=0.85 ; p<0 \cdot 001)$ (Fig. 5). Administration of pentagastrin decreased the resistivity in every case except one and the maximum change in resistivity that occurred after pentagastrin ( $R$ maximum) was correlated with the maximal acid output $(r=0.58$; $\mathrm{p}<0 \cdot 05)$ (Fig. 5).

EFFECT OF ALCOHOL.

Ingestion of alcohol produced a gradual change in gastric resistivity in all six subjects (Fig. 6). The maximum change was seen approximately 25-30 minutes after administration of alcohol and was about $50 \%$ of that observed after a drink of Oxo by the same subject. The values then returned towards basal levels. Administration of cimetidine one and a half hours before the study abolished the change in resistivity induced by alcohol (Fig. 6).

\section{Discussion}

The aim of these studies was to determine whether applied potential tomography could be used to provide a non-invasive index of gastric acid secretion.

Results in volunteers showed APT responds to the volume and hydrogen ion concentration of acid solutions introduced into the stomach, although changes in gastric resistivity are more closely correlated with the overall conductivity of the gastric contents. Because hydrogen ions would make a larger contribution to conductivity than other ions on account of their greater ionic mobility, APT should provide a useful index of acid output under most physiological conditions.

Studies carried out in patients showed significant correlations between changes in gastric resistivity before and after pentagastrin and measurements of acid output conducted on a separate occasion. The fact that the correlations were not stronger may be explained by a number of factors. First, there were necessary differences in methodology between APT and aspiration studies (presence of a tube and continuous aspiration of gastric contents) which may have affected the amount of acid secreted on the two occasions. Second, spuriously high values (suggesting acid secretion) could theoretically be obtained using APT if large amounts of sodium rich duodenal juice reflux into a stomach that is secreting small amounts of acid. Third, gastric emptying is most commonly ignored in aspiration studies as gastric juice is being continuously removed, however, it may 


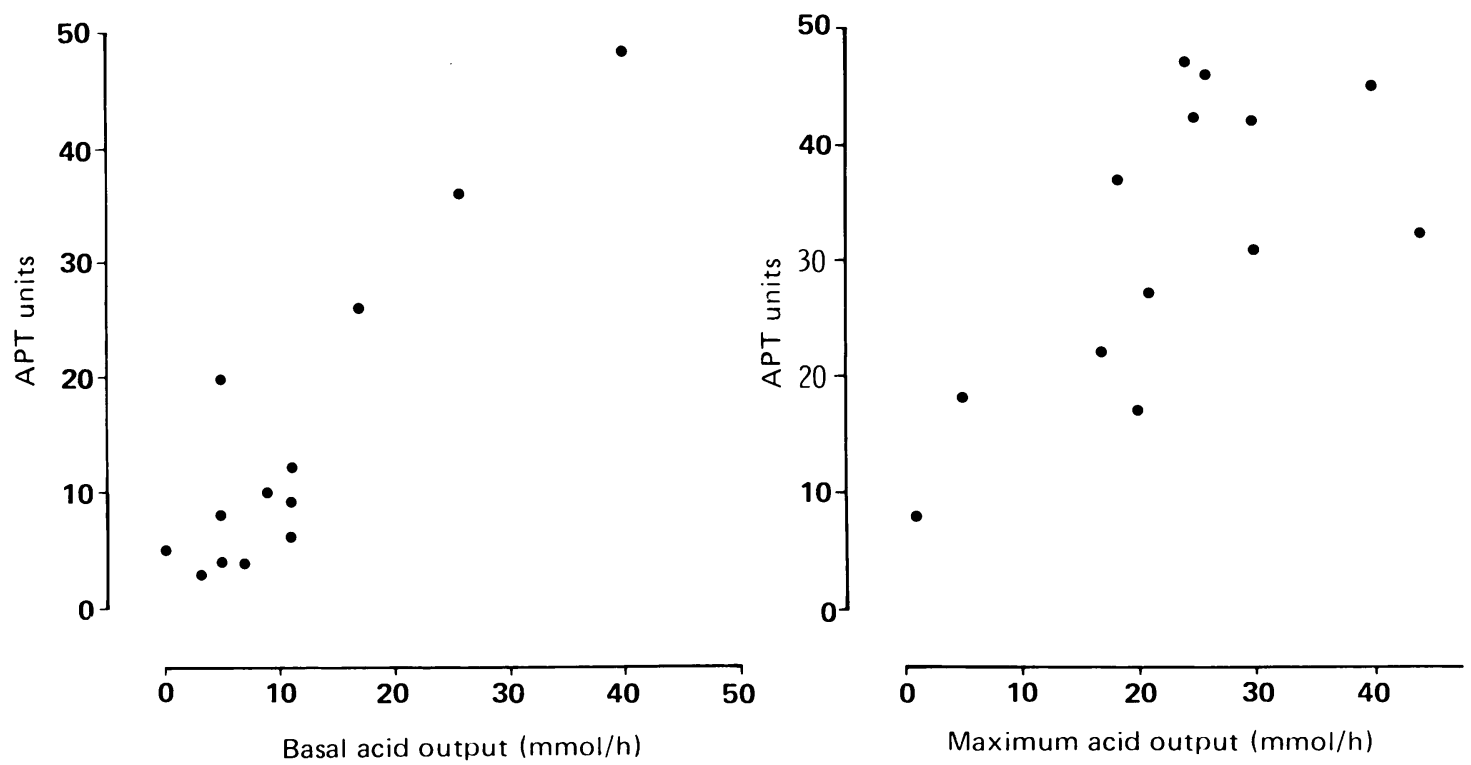

Fig. 5 Relationship between changes in resistivity observed in 13 patients before pentagastrin injection $(R$ basal) and basal acid output and the changes observed after pentagastrin ( $R$ maximum) and maximum acid output.

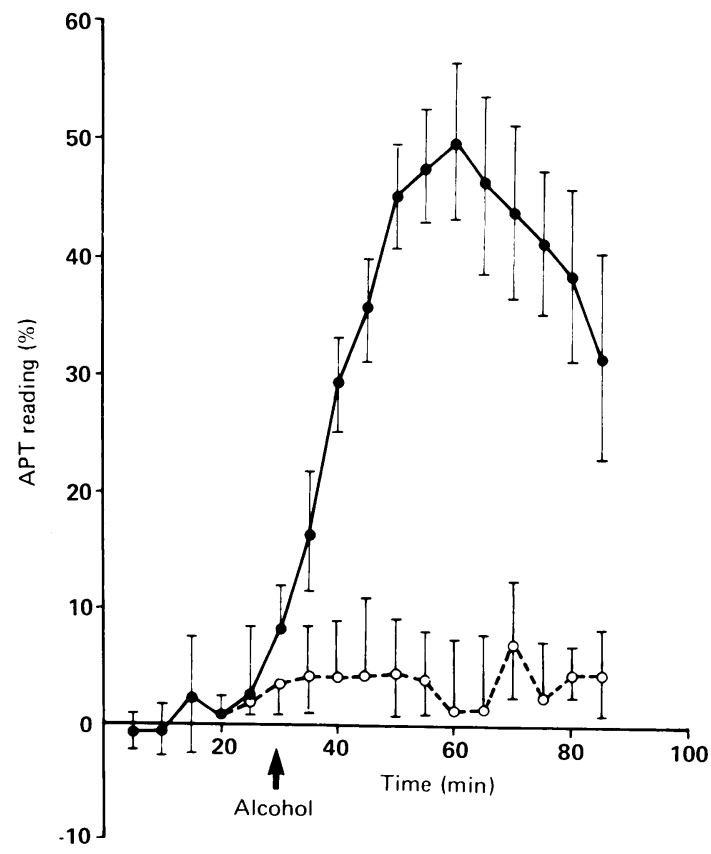

Fig. 6 Changes in gastric resistivity in six fasted volunteers after a drink of sherry. The results (mean (SE)) are expressed as a percentage of the value obtained after a drink of $300 \mathrm{ml}$ Oxo. Dotted lines indicated readings obtained in three volunteers after administration of cimetidine. be more significant when acid output is measured by APT, as the acid is allowed to accumulate. The loss of acid from the stomach will lower the estimated amount of acid secreted. Conductivity measurements during the basal half hour did fluctuate, indicating that gastric emptying may be significant. While it is possible to measure loss of gastric acid from the stomach by dye dilution techniques and also to measure duodenal reflux by flame photometry, it was not done in this study as we wished to investigate the usefulness of APT as a non-invasive index of acid secretion.

Applied potential tomography is a non-invasive technique with no known contra-indications or unwanted effects. It is preferred by patients, is simple to operate, and though more costly than the equipment used in a pentagastrin test, it can also be used in other measurements, such as gastric emptying" and lung function. ." Our results suggest that APT may also be sufficiently accurate to initially screen patients for suspected gastrinomas and achlorhydria, one of the main uses of the pentagastrin test in our department. The most suitable application of APT would, however, be in providing continuous monitoring of acid secretion in intensive care or postoperative patients. As a research tool, it could allow the secretory response to such factors as alcohol, smoking, sham feeding, or stress to be assessed without the modulating influence and discomfort of a gastric tube. 


\section{References}

1 Baron JH. Clinical tests of gastric secretion. London: MacMillan Press, 1978: 5-6.

2 Segal HL, Miller LL, Morton JJ. Determination of gastric acidity without intubation by the use of cation exchange indicator compounds. Proc Soc Exp Biol Med 1950; 4: 218-20.

3 Bock OAA. Witts LJ. Tubeless gastric analysis with Azure A and betazole hydrochloride. Br Med J 1961; ii: 665-7.

4 Knapton PJ. Hawkins CF, Whitehead TP. Tubeless gastric analysis. Trial of the gastro test. Lancet 1964; i: 439.

5 Taylor TV, Bone D, Torrance B. A non-invasive test for gastric function. Br J Surg 1977; 64: 702-8.

6 Taylor TV, Holt S, McLoughlin GP, Heading RC. A single scan technique for estimating acid output. Gastroenterology 1979; 77: 1241-4.
7 Smith L, Corinaldesi R, Casadio R, Williams JG. Scanning for acid. Gastroenterology 1980; 79: 608 .

8 Brown BH. Barber D. Freeston IL. UK Patent No. 2119520. Potential tomography, priority. April 1982.

9 Barber DC, Brown DH. Applied potential tomography. J Phys E Sci Instrum 1984; 17: 723-33.

10 Brown BH, Barber DC, Seagar AD. Applied potential tomography: possible clinical applications. Clin Phys Physiol Meas 1985; 6 2: 109-21.

11 Avill R, Mangnall YF, Bird NC, et al. Applied potential tomography: a new non-invasive technique for measuring gastric emptying. Gastroenterology 1987: 92: 101926.

12 Harris ND, Suggett AJ, Barber DC, Brown BH. Applications of applied potential tomography in respiratory medicine. Clin Phys Physiol Meas 1987; 8 (suppl A): 155-6.5. 